(Chinese identification system) on each definition. Results: The prevalence of healthy aging increases when the operational definitions are loosened, ranging from 3.9\% (most restrictive definition) to $15.2 \%$ (least restrictive). Across definitions, the odds of healthy aging were lower for people of advanced age, low education, female gender, and without pension when compared to their counterparts. Discussion: Our findings regarding prevalence of healthy aging helps to set a benchmark to plan and evaluate initiatives to promote healthy aging in China. Our preliminary findings showed that disability prevalence was higher than that reported in a prior U.S. study. A reason for the difference is that our sample was less likely to have major diseases but more likely to be disabled compared to the U.S. sample. This suggests that the reporting of disease and the path from disease to disability may be different between older adults in China and the U.S. However, socioeconomic disadvantages associated with healthy aging are similarly supported in our and the U.S. study.

\section{COMPARING SUCCESSFUL AGING, RESILIENCE, AND HOLISTIC WELLNESS AS PREDICTORS OF THE GOOD LIFE}

M. Fullen ${ }^{1}$, V. Richardson ${ }^{2}$, 1. Virginia Tech, 2. The Ohio

State University

There is ongoing debate over how to define successful aging. Historically, biomedical conceptualizations of older adulthood have prevailed. Recently, gerontologists have criticized the theory of successful aging, describing it as exclusive and overly emphasizing physical and functional capacities (Martinson \& Berridge, 2015). In its place, scholars have recommended alternative frameworks such as resilience (Jeste et al., 2013) or holistic wellness (Strout et al., 2016), arguing that these frameworks are more responsive to the heterogeneity inherent among older persons. The purpose of our study was to compare the relative contributions of Rowe and Kahn's definition of successful aging (SA), resilience, and the holistic wellness paradigm for predicting happiness, life satisfaction, and self-rated physical health. We sampled adults residing in independent living senior housing communities. We hypothesized that in each of three full regression models, holistic wellness and resilience would be significant predictors of happiness, life satisfaction, and physical health, whereas successful aging status would not be a significant predictor. In sum, the criteria underlying successful aging poorly predicted happiness, life satisfaction, and self-rated physical health compared to the resilience and holistic wellness models. When predicting happiness and life satisfaction, race and holistic wellness were significant predictors. Age and holistic wellness were the best predictors of self-rated physical health. The results suggest that definitions of aging well are complex and require greater nuance. The findings have important implications for clinicians seeking translatable theoretical models that are amenable to practice with older adults, especially for those living in independent senior housing communities.

\section{CONTROL OVERRIDES ETHNICITY IN PREDICTING A POSITIVE VIEW OF AGING}

M. Clark, Queens University of Charlotte
Attitudes toward aging have been shown to be related to both quality of aging and mortality. Those with better attitudes are happier in old age and live longer than those with more negative attitudes. Research has attempted to identify factors that affect attitudes toward aging; many focusing on ethnic differences. However, simple differences may mask a more complicated relationship between ethnicity and attitudes toward aging. This paper (part of a larger study of 1875 participants, ages 18-99) presents data from 1447 participants who answered whether old age is seen as a happy time. The sample included 306 African Americans, 219 Asian Americans, 620 White Americans, 142 Hispanic Americans, 51 Middle Eastern Americans, 41American Indians, and 68 who identified as Multiracial. Significant ethnic differences were seen in viewing aging as happy, X2 $(12, \mathrm{~N}=1253)=22.67, \mathrm{p}=.03$. However a multiple linear regression was conducted including a 3 part measure of control (shown to affect both attitude and mortality) and ethnicity as predictors of whether or not old age is seen as happy. In this analysis, ethnicity failed to predict attitude but instead, control by powerful others predicted attitude, $\mathrm{F}(1,1032)=55.451, \mathrm{p}<.001 ; \mathrm{R} 2=.051$. Additional analyses showed that fear of aging was also not predicted by ethnicity (despite significant differences). More detailed data will be presented to show the more complex relationship between ethnicity and attitudes towards aging. If education programs are to be effective, they must incorporate this more nuanced association.

\section{DISCOURSES OF BISEXUALITY AMONG OLDER WOMEN}

S. Jen, University of Washington

Lesbian, gay, bisexual, transgender, and queer (LGBTQ) older adults are an understudied and underserved population. However, subgroup differences are often overlooked including the lives of older bisexual women who experience significant disparities in health. The historical and sociocultural context around bisexuality of older adults is one unexamined factor that may influence the ability of older bisexuals to achieve equitable health outcomes. To address this gap, this study applied a life course perspective and Foucauldian Discourse Analysis method to analyze how bisexual women $(\mathrm{N}=13)$ age 60 and older construct their bisexual identities and subjectivity through language use and by drawing on or creating broader discourses within their particular historical context. Findings indicate a tension between constructing bisexual identities as freedom, potential, or possibility versus the negative effect this identity has had on participants' social, personal, and political resources over the life course. This tension is evident in participant's narratives, as some describe bisexuality as a source of pride while others report a deep sense of ambivalence toward their bisexual identity. Both of these constructions are informed by the historical context in which participants' recognized their attractions to women and experienced the coming out process. These tensions and differences in experience have implications for the ability of researchers to interpret how identity-related factors are relevant to and influence the health and wellbeing of older bisexual women as well as for practitioners who seek to provide culturally competent services to older bisexual women. 